\title{
Phase Transitions in Ion-Exchange Materials during the Water Vapor Sorption
}

\author{
Igor Ivanov ${ }^{1, *}$ and Oksana Ivanova ${ }^{2}$ \\ ${ }^{1}$ Russian State Agrarian University, Kaluga branch, RU-248007, Kaluga, Russia \\ ${ }^{2}$ Moscow State Technological University "STANKIN", RU-127055, Moscow, Russia
}

\begin{abstract}
Water vapor sorption process on ion-exchange materials was investigated using a model taking into account the mutual influence of variations in hydration energy and osmotic effect. At realistic parameter values, phase transitions of the first kind of the thin adsorbed liquid film and thick adsorbed liquid film type can occur. Possible reasons and criteria of the phase transitions that led to stepwise change of ionite resistance were discussed. The phase transitions obtained in this work manifest themselves as hysteresis loops on water vapor adsorption-desorption experimental isotherms. Hysteresis was observed experimentally both at high and at low values of relative air humidity. It was explained using different models: for high and low humidity separately. The present work made it possible to explain these critical phenomena using single mathematical model.
\end{abstract}

\section{Introduction}

An adequate description of the water vapor sorption by ion-exchange materials (ionites or ion exchangers (IEs)) is possible only on the basis of models that take into account the specific feature of IEs: the relationship between the amount of absorbed water and the dissociation of ion-exchange groups (ionogenic groups (IGs)) [1-8]. On the one hand, an increase in water content enhances hydration and, consequently, dissociation of IGs. On the other hand, an osmotic effect takes place [3], i.e. the dependence of the moisture content on the degree of dissociation. In the presence of such a mutual dependence, a situation is possible when a fluctuating increase in moisture content enhances the dissociation of IGs, which, due to the osmotic effect, leads to the condensation of additional water molecules into the IE phase. As a result, under certain conditions, fluctuations greater than a certain value will not decay, but grow until the system enters a new state. This state is characterized by a moisture content and degree of dissociation significantly different from the initial ones, so this transition can be considered as a phase transition of the first kind, similar to the phase transitions of a thin film - a thick film of an adsorbed substance [9] or a strong electrolyte - a weak electrolyte [10]. Since a stepwise change in the dissociation degree leads to a corresponding change in the conductivity of IE, the phase transitions can be observed experimentally in form of a stepwise change in IE sample resistance.

In work [5], based on a model that takes into account the mutual influence of variations in hydration energy and osmotic effect, the possibility of phase transitions of the thin adsorbed liquid film and thick adsorbed liquid film type for high humidity $(0,6<\theta<0,8)$ was shown.

Based on thermodynamic approach consistently developed in $[5,11,12]$, the possibility of phase transitions during the water vapor sorption in IEs over the entire range of relative air humidity has been investigated in the present work. Given model made it possible to refine the phase transition criterion obtained in [5] and to find the phase transition at low humidity $(\theta<0,3)$ at physically reasonable values of system parameters. The adequacy of these parameters is substantiated by comparing the theoretical and experimental dependences of the sulfocationite membranes samples resistance on the relative air humidity. This approach allows one to obtain basic (noncritical) system parameters at which a phase transition is impossible. And the sequential change of these parameters makes it possible to simulate a phase transition and find out the criterion for its occurrence. The parameters obtained in this way will be critical, and the relationship between them will be phase transition criterion.

As a result of mathematical modeling, the critical dependences of the resistance of IEs samples on the relative air humidity were obtained. These dependences have an $S$-shaped character, typical for systems with a phase transition of the first kind. We note once again that the study of the phase transitions on the above dependences is equivalent to the study of this phenomenon on the adsorption isotherms (the dependence of the moisture content on the relative air humidity): due to the mutual dependence of the degree of IGs dissociation and the moisture content, the phase transition also manifest itself in a stepwise change in the 
concentration of free ions in solution, and, therefore, in the corresponding change in the IE sample resistance.

The phase transitions obtained in this work manifest themselves as hysteresis loops on water vapor adsorption-desorption experimental isotherms. Hysteresis was observed experimentally both at high and at low values of relative air humidity [13]. It was explained using different models: for high and low humidity separately. The present work made it possible to explain these critical phenomena using single mathematical model.

\section{Theoretical Analysis}

Mathematical model of the water vapor sorption in IEs was built by minimizing the total thermodynamic potential of the system. This potential consists of the thermodynamic potential of the ionite in the standard state, which was defined as the state in which the ionite contains only the water entering into the hydration shells; the change in the thermodynamic potential caused by the subsequent condensation of water; the ionic subsystem potential, more specifically, this associated with the dissociation of IGs, the formation of their hydration shells, and the formation of a solution composed of a hydrated ions and free water, as well as the entropy contribution associated with the different configurations in the positioning of dissociated and nondissociated IGs in the polymer matrix; and finally the potential associated with elastic deformation of the polymer chains during moisture sorption. In comparison with previous models, this model calculates the IGs dissociation energy more precisely. In addition to the effects of the surface charge and the Debye screening, the ion-dipole interaction during the sorption of polarized molecules is taken into account. The contribution of these factors is most significant at low humidity.

Without going into details of calculation described in work [12], we present the system of equations describing water vapor sorption on the surface of IE:

$$
\left\{\begin{array}{l}
P / P_{S}=\frac{\beta-\beta_{0}-\alpha n}{1+\beta-\beta_{0}-\alpha(n-1)} \exp \left(\frac{\alpha}{T} \frac{\partial E_{D}}{\partial \beta}+U \beta\right), \\
\frac{\alpha^{2}}{1-\alpha} \frac{\left(1+\beta-\beta_{0}-\alpha(n-1)\right)^{n-1}}{\left(\beta-\beta_{0}-\alpha n\right)^{n}}=\exp \left(-\frac{1}{T} \frac{\partial\left(\alpha E_{D}\right)}{\partial \alpha}\right) .
\end{array}\right.
$$

The system includes the following parameters: $P / P_{s}=\theta$ is the relative air humidity, $P_{s}$ is the saturation vapor pressure; $\beta=N_{W} / N$ is the relative moisture content, $\beta_{0}=N_{W 0} / N$ is the conventional number of hydration of IGs, were $N$ is the number of IGs bonded to the polymer matrix, $N_{W 0}$ is the number of water molecules entering into the hydration shells of the IGs, and $N_{W}$ is the total number of water molecules in the system; $\alpha$ is the degree of dissociation of IGs; $n$ is the conventional number of hydration of ions in a singlecomponent solution; $T$ is the absolute temperature expressed in energy units; $E_{D}=E_{0}+E_{E L}$ is the dissociation energy, which consists of the energy of abstraction of the ion from the polymer chain $\left(E_{0}\right)$ and the change in its electrostatic energy $\left(E_{E L}\right)$; $U \beta=1 / 2 \partial\left(U \beta^{2}\right) / \partial \beta$, were $U T \beta^{2} / 2$ is the energy of elastic deformation of the polymer chains per IG, $U=6\left(V_{W} / V_{P}\right)^{2}, V_{W}$ is the molecular volume of water, and $V_{P}$ is the polymer chain volume per IG.

Passage to the limits transforms this system into standard expressions: at large values of the relative moisture content $\partial\left(\alpha E_{D}\right) / \partial \alpha \rightarrow E_{0}, \partial E_{D} / \partial \beta \rightarrow 0$, and, therefore, formula (1) transforms into the standard expression for the concentration dependence of the vapor pressure over solution [14], while formula (2) takes the form of the law of mass action [15]. Formulas (1) and (2) make it possible not only to determine the values of $\alpha$ and $\theta$ for the bulk phase, but also to examine how they change in the case of sorbed thin water films.

As shown in work [12], the dependence of the energy $E_{D}$ on the degree of dissociation $\alpha$ and the water content $\beta$ takes the form

$$
\begin{gathered}
E_{D}=E_{0}-\frac{C T}{\beta}\left(\frac{\xi \sqrt{\alpha \beta}}{2}+\ln (1-f \exp (-\xi \sqrt{\alpha \beta}))\right) \\
-\frac{\xi T \sqrt{\alpha \beta} \exp (-\xi \sqrt{\alpha \beta} / 2)}{1-\exp (-\xi \sqrt{\alpha \beta})}-\frac{n \delta}{12^{1 / 3}}(C \xi)^{2 / 3} T,
\end{gathered}
$$

where $C=2 q^{2} s /\left(\varepsilon T V_{W}\right)$ and $\xi=\left[4 \pi q^{2} V_{W} /\left(\varepsilon T s^{2}\right)\right]^{1 / 2}$ are dimensionless quantities that include the following parameters: $q$ is the ion charge, $s$ is the surface area per IG, $\varepsilon$ is the dielectric permittivity of the solution; $f=\left(\varepsilon-\varepsilon_{1}\right) /\left(\varepsilon+\varepsilon_{1}\right), \quad \varepsilon_{1}$ is the dielectric permittivity of the polymer matrix; $\delta q$ is effective charge of dipole edges (polarized water molecule $\delta \sim 0,1$ ).

The terms in (3), starting from the second, correspond to the contributions to the electrostatic energy from the bulk of the screening cloud and the charges induced in the solid dielectric layers due to the finite thickness of the liquid layer, the charge of the surface, and the ion-dipole interaction respectively.

The expression (3) is valid at all physically reasonable values of system parameters (water content and degree of dissociation), even at lowest humidity, when hydration shells of ions are not formed completely (the dependence $n(\beta)$ is needed). For this reason, this expression can be used over the full relative humidity range.

Solving the system of equations (1), (2) with consideration given to (3) makes it possible to determine the dependence of the relative moisture content $\beta$ on the relative humidity $\theta$, i.e. adsorption isotherm $\beta(\theta)$, and the dependence of the degree of dissociation $\alpha$ on the relative humidity $\theta$, i.e. $\alpha(\theta)$. In terms of searching for phase transitions, these dependences are equivalent: hysteresis is shown on each of them. Since we have experimental data of dependences of the resistance of IE sample $R$ on the relative humidity $\theta$ at various temperatures [12], we consider it appropriate to investigate the theoretical dependences $R(\theta)$.

To describe the conduction in IEs, which are nonuniform media, we used the concepts underlying the 
theory of percolation [16]. According to [16], the current flowing through a nonuniform medium is determined by the lowest conductivity areas on the route between the electrodes of the measuring unit. Conductivity areas look like channels connecting the electrodes. Water molecules are adsorbed in these channels that leads to channel water-filling, IGs dissociation, and appearance of a current between the electrodes. Thus, it is sufficient to calculate the conductivity of these areas.

To calculate the resistance of the system from the degree of dissociation $\alpha$, we assumed that the mobility of the ions $\mu$ is humidity-independent, i.e. the conductance $\lambda$ depends only on concentration $c$ of free carriers. Then the resistance of IE sample

$$
R=\frac{1}{\lambda} \frac{l}{S}=\frac{1}{\mu q c} \frac{l}{S},
$$

where $l$ is the characteristic length of conducting channel between electrodes and $S$ is the summary cross section area of all conducting channels.

Considered that the concentration of free ions in solution $c=\alpha N / S l$ we obtained

$$
R=\frac{l^{2}}{\mu q N} \frac{1}{\alpha}=\frac{R_{0}}{\alpha},
$$

where parameter $R_{0}=l^{2} /(\mu q N)$ depends on the geometry of the sample and the concentration of active IGs but does not depend on the humidity.

The empirical temperature dependence of the ion mobility $\mu$ can be presented as [17]

$$
\mu=\mu_{0}\left(1+\gamma\left(t-t_{0}\right)\right),
$$

where $\gamma \approx 0,1$ and $t_{0}=15{ }^{\circ} \mathrm{C}$.

Taking into account formulas (3), (4), and (5), let us proceed to the analysis of equations system (1) and (2) for the possibility of phase transitions. As follows from these equations, the effect of changing the hydration energy $E_{E L}$ with moisture content $\beta$ increasing is determined by the value of the coefficient $C$. At low values of $C$, the change in hydration energy is insignificant. With the growth of parameter $C$, the mutual influence of hydration and osmotic effect increases, and unstable states and phase transitions can occur. Obviously, the criterion of a phase transition is the existence of such $\theta$ values at which $d R / d \theta \rightarrow \infty$. Let's try to get the "extreme" values of the system parameters, i.e. such when phase transition are still possible.

\section{Results and Discussion}

The analytical study of $R(\theta)$ dependence is rather cumbersome. Therefore, in general, for arbitrary dissociation degrees, the answer to the question of the possibility of phase transitions can be obtained only on the basis of a numerical solution of the system (1) and (2). At first, the "basic" (noncritical) dependences were plotted at different temperatures (Fig. 1, (1)). These dependences were calculated using the following values of the model parameters: $q=4,8 \cdot 10^{-10}$ esu, $\varepsilon=79$, $\varepsilon_{1}=4, \quad V_{W}=3 \cdot 10^{-23} \mathrm{~cm}^{3}, \quad s=1,2 \cdot 10^{-15} \mathrm{~cm}^{2}, \quad l=1 \mathrm{~cm}$, $n=4, \quad$ and $\quad U=1 ; \quad$ at $t=t_{0}=15^{\circ} \mathrm{C}, \quad \mu_{0}=3,2 \cdot 10^{-}$ ${ }^{4} \mathrm{~cm}^{2} /(\mathrm{V} \cdot \mathrm{s}), \quad K_{0}=1,2$, and $R_{0}=2 \mathrm{k} \Omega$. The elastic deformation energy parameter $U$ was assumed to be temperature-independent. The circles represent the experiment data obtained on the sulfocationite membranes samples [12]. It should be noted that a good agreement between theoretical and experimental values at quite realistic system parameters confirms the adequacy of the proposed model. By successively increasing the value of parameter $C$, it is possible to obtain critical dependences (Fig. 1, (2), (3)) with pronounced areas where $d R / d \theta \rightarrow \infty$, corresponding to the phase transitions beginning. A further increase in the parameter $C$ leads to the appearance of characteristic $S$ shaped curve pieces (Fig. 2, (2), (3)), which are shown as stepwise sections or hysteresis loops on an experimental curves. Indeed, like other systems in which phase transitions of the first kind are possible, the transition to a new phase occurs when fluctuations exceed a certain critical value [15]. The probability of such fluctuations is usually quite small that can practically mean the existence of long-lived metastable states. As a result, in experiment the transition from the thin film (weak electrolyte) phase to the thick film (strong electrolyte) phase can occur at humidity different from that at which the reverse transition is observed (i.e. hysteresis on water vapor adsorption-desorption isotherms can be an experimental manifestation of the phase transitions obtained in the present work).

Thus, the numerical analysis of equations (1) and (2) gives rise to the following criterion of phase transition:

$$
C \geq 4,7 \beta_{0},
$$

where numerical coefficient before $\beta_{0}$ is slightly decreased with $n$ reduction (if $n=1$ this coefficient equals 4,1), so that it can be set constant.

In addition the present model explains phase transitions appearance both at high and at low humidity values. For example, such situation can appear in ionites with a small number of hydration of surface IGs $\left(\beta_{0}=1\right.$, Fig. 2, (2)) or with rarely placed IGs (large surface area $s$ per IG, Fig. 2, (3)). Therefore, it is necessary to take into account these facts in measurements of IEs conductance. During the manufacturing of hygrometers with IEs sensors, phase transitions should be avoided by selecting proper materials with noncritical parameters (Fig. 2, (1)).

According to [13], the water vapor adsorption hysteresis on sulfocationite membranes was discovered by some authors at high humidity $(0,6<\theta<0,8)$. Other authors discovered the hysteresis at low humidity $(\theta \leq 0,3)$. Note, these experimental results are in close agreement with the results of the present work (as can be seen from Fig. 2, dependence 3 has hysteresis at high humidity and dependence $2-$ at low). These authors explain the appearance of hysteresis as a result of capillary condensation. The present model explains the hysteresis by correlation between the water content and 
the dissociation of IGs: the fluctuating increase of water content leads to increase of IGs dissociation and, because of osmotic effect, the additional water molecules come into ionite. Moreover, this model explains the hysteresis at low humidity, when the capillary condensation is impossible in principle.

Thus, the results of the present work show that using a single model, we can theoretically explain the experimentally observed phase transitions both at high and at low humidity [13].

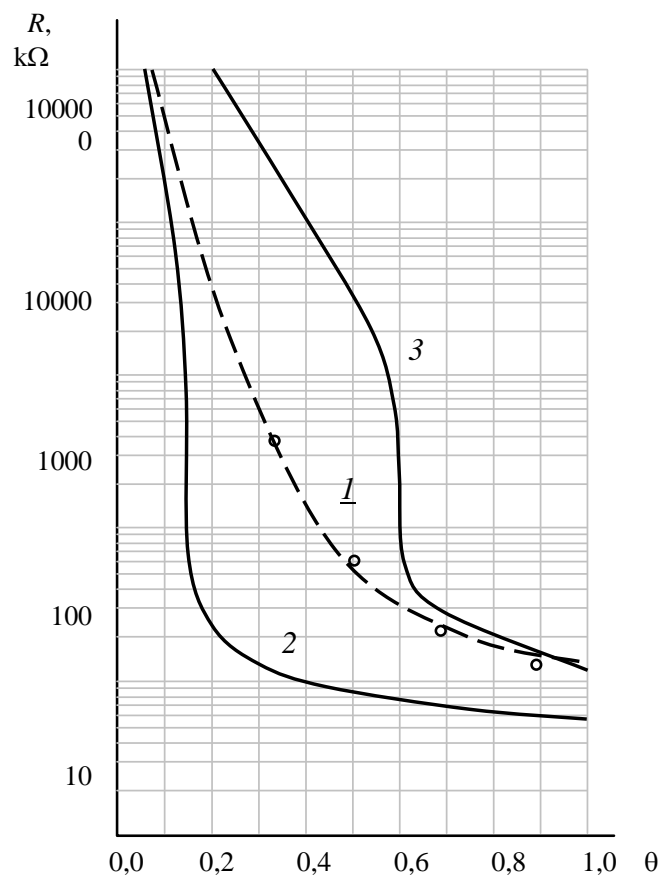

(a)

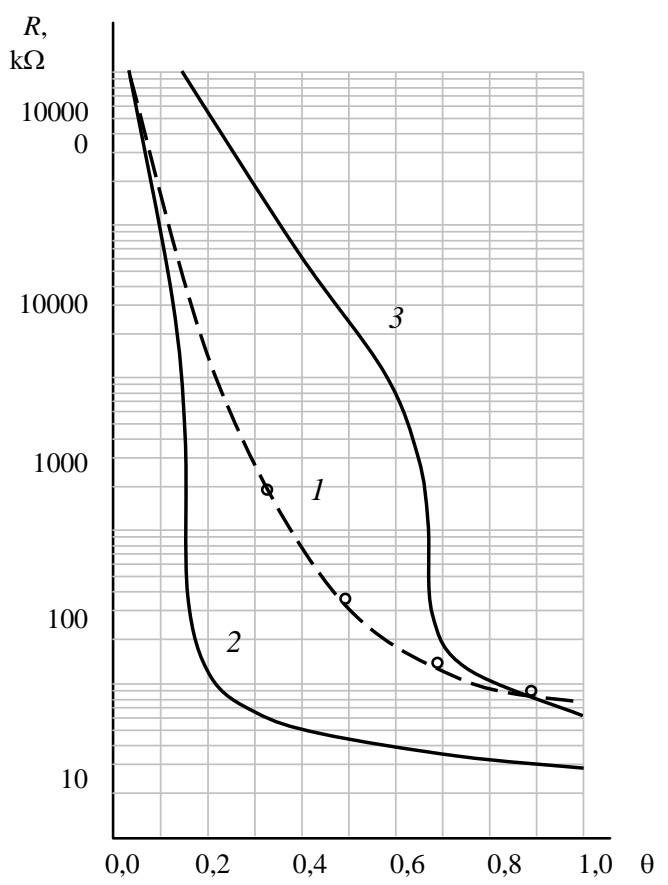

(c)

\section{Conclusion}

Obviously, in order to obtain more accurate quantitative results for real systems, the proposed calculation method can be somewhat refined or modified. For this purpose it is possible to consider details of adsorption of water molecules specifically interacting with active centers [3], dependence of dissociation energy on geometry of growing clusters [18], effect of self-dissociation of electrolyte $[10,15]$. However, these effects will slightly

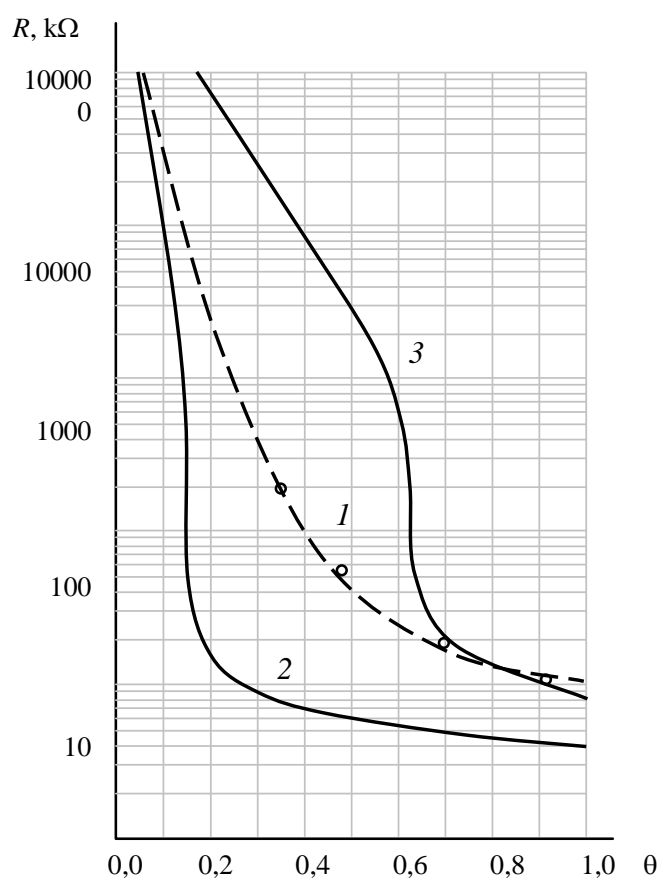

(b)

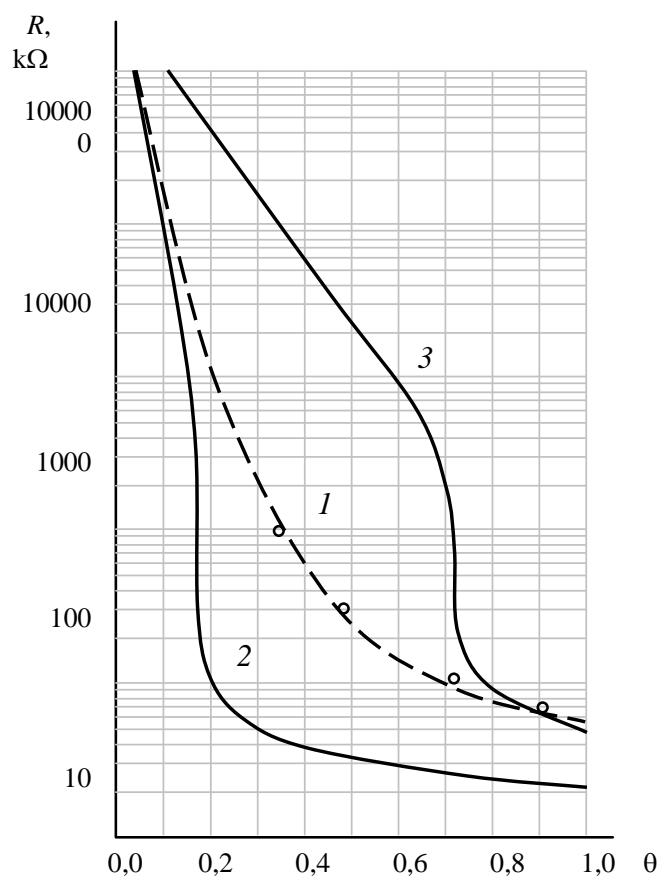

(d)

Fig. 1. The $R(\theta)$ dependence at various temperatures:

(a) $t=15^{\circ} \mathrm{C}, \quad \beta_{0}=2, C=5,87$ (1), $\beta_{0}=1, C=4,65$ (2), $\beta_{0}=2, C=9,54$ (3);

(b) $t=20^{\circ} \mathrm{C}, \quad \beta_{0}=2, C=5,77$ (1), $\quad \beta_{0}=1, C=4,67$ (2), $\quad \beta_{0}=2, C=9,57$ (3);

(c) $t=30^{\circ} \mathrm{C}, \quad \beta_{0}=2, C=5,58(1), \quad \beta_{0}=1, C=4,69$ (2), $\beta_{0}=2, C=9,67$ (3);

(d) $t=40^{\circ} \mathrm{C}, \quad \beta_{0}=2, C=5,40$ (1), $\quad \beta_{n}=1, C=4,70$ (2), $\beta_{0}=2, C=9,77$ (3). 


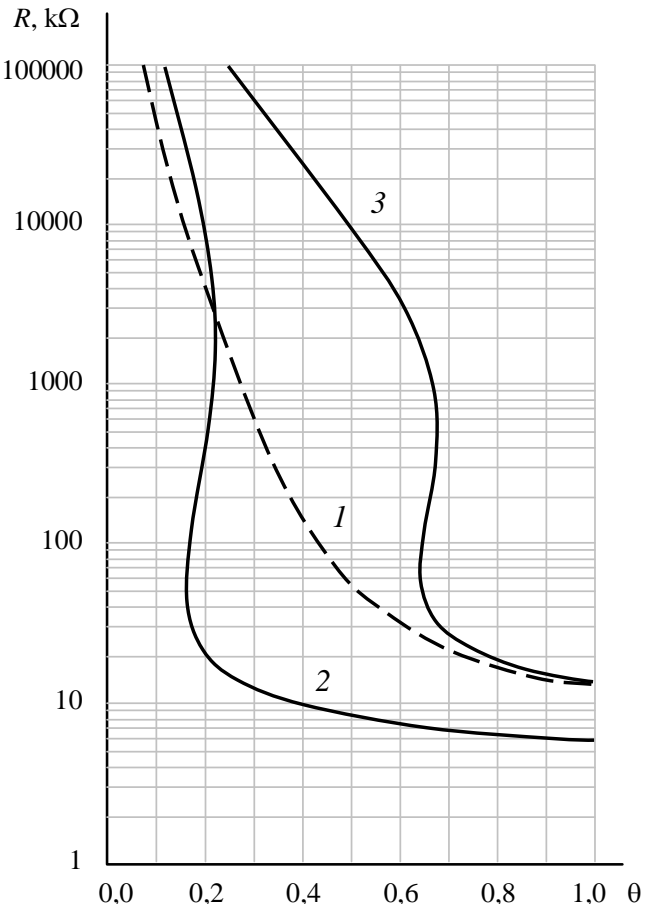

Fig. 2. The $R(\theta)$ dependences at $\beta_{0}=2, C=5,87$ (1); $\beta_{0}=1, C=5,87$ (2); and $\beta_{0}=2, C=10,27$ (3).

change the quantitative parameters of adsorption and will not affect the overall physical picture of the occurrence of phase transitions proposed in the present work.

The question of the possibility of experimental observation of the phenomenon described in the present work at high humidity, when capillary condensation is possible, remains open. The fact is that the effects of capillary condensation also manifest themselves in steps and hysteresis loops on adsorption isotherms and the dependences of the resistance of IEs samples on the relative air humidity. The contribution of these two phenomena can be unambiguously estimated by making measurements according to the following scheme. At first, the adsorption isotherms are measured on some porous matrix. After that, the surface of the pores is covered with IGs by precipitation from a condensed solution, and after drying, the adsorption isotherms are measured. The new loops and steps arising in this case can be attributed to the phenomenon described in this work. Another possible method is the study of weakly crosslinked polymer matrices where the appearance of pores with rigid walls, and, consequently, capillary condensation, seems unlikely. Therefore, the hysteresis loops observed at $\theta \sim 0,6-0,8$ in such materials can also be attributed to this phenomenon.

In conclusion, it should be noted that the obtained results force us to be cautious about the possibility of using capillary condensation methods in IEs porosimetry [19]. For example, when condition (6) is satisfied, a hysteresis loop, as shown in the present work, can be observed even during adsorption on a smooth surface. At the same time, if we assume that its appearance is due to capillary condensation, we can get the wrong conclusion about the existence of pores of a sufficiently small radius in the system. We emphasize, however, that if an IE contains closely placed IGs $\left(C<4,7 \beta_{0}\right)$, condition (6) is violated, and the anomalies observed in this case can be unambiguously attributed to the effect of capillary condensation.

\section{References}

1. Y. Tanaka, Ion Exchange Membranes: Fundamentals and Applications (Elsevier, Tokyo, 2015)

2. Yu.A. Kokotov, Ion Exchangers and Ion Exchange (Khimiya, Leningrad, 1980)

3. A.I. Gantman, Zh. Fiz. Khim., 66, 1713 (1992) [in Russia]

4. B. Conway, Ionic Hydration in Chemistry and Biophysics (Elsevier, Amsterdam, 1981)

5. V.M. Agafonov, I.V. Ivanov, Russ. J. Phys. Chem., 70, 825 (1996)

6. V.I. Vasil'eva, E.M. Akberova, V.A. Shaposhnik, M.D. Malykin, Russ. J. Electrochem., 50, 789 (2014)

7. V. Soldatov, V. Zelenkovskii, E. Kosandrovich, React. Funct. Polym., 102, 147 (2016)

8. N. Kononenko, V. Nikonenko, M. Fomenko, D. Grande, C. Larchet, L. Dammak, Y. Volfkovich, Adv. Colloid Interfac., 246, 196 (2017)

9. J. Cahn, J. Chem. Phys., 66, 3667 (1977)

10. V.A. Kozlov, S.V. Sokolova, N.A. Trufanov, Zh. Eksp. Teor. Fiz., 98, 2176 (1990) [in Russia]

11. I.V. Ivanov, Russ. J. Phys. Chem., 79, 448 (2005)

12. I. Ivanov, O. Ivanova, EPJ Web Conf., 224, 03001 (2019)

13. Yu.A. Kokotov, V.A. Pasechnik, Equilibrium and Kinetic of Ion Exchange (Khimiya, Leningrad, 1970)

14. L.D. Landau, E.M. Lifshits, Course of Theoretical Physics, Vol. 5: Statistical Physics, 2nd ed. (Nauka, Moscow, 1964; Pergamon, Oxford, 1980)

15. Ya.I. Frenkel', Kinetic Theory of Liquids (Nauka, Leningrad, 1975; Clarendon, Oxford, 1946)

16. S.F. Timashev, S.N. Cladkikh, Dokl. Akad. Nauk SSSR, 262, 656 (1982)

17. L.I. Antropov, Theoretical Electrochemistry (Vysshaya Shkola, Moscow, 1975)

18. R.R. Dogonadze, F.F. Kornyshev, A.M. Kuznetsov, Teor. Mat. Fiz., 15, 127 (1973)

19. S. Gregg, K. Sing, Adsorption, Surface Area and Porosity (Academic Press, London, 1982) 